\title{
Running Form Analysis Based on Impact Dynamics: A Minimally Complex Mechanical Model
}

\author{
László Bencsik ${ }^{*}$, Ambrus Zelei ${ }^{2}$ \\ ${ }^{1}$ MTA-BME Lendület Human Balancing Research Group, Department of Applied Mechanics, Faculty of Mechanical Engineering, \\ Budapest University of Technology and Economics, 1111 Budapest, Műegyetem rkp. 5., Hungary \\ 2 MTA-BME Research Group on Dynamics and Machines of Vehicles, Department of Applied Mechanics, Faculty of Mechanical \\ Engineering, Budapest University of Technology and Economics, 1111 Budapest, Múegyetem rkp. 5., Hungary \\ * Corresponding author, e-mail: bencsik@mm.bme.hu
}

Received: 28 November 2017, Accepted: 04 October 2018, Published online: 05 November 2018

\begin{abstract}
Biomechanical models of different complexity are used to understand the dynamics of human running. Low degrees-of-freedom models are appropriate for the prediction of the effect of certain parameter changes. We present a minimally complex biomechanical model which characterizes the effects of foot strike pattern and shank angle on the ground-foot impact intensity, which influences the risk of injuries and energy efficiency.

A three segment leg model (thigh, shank and foot) is proposed combined with the mass of the rest of the body parts concentrated in the hip. The ground-foot impact intensity and the absorbed kinetic energy are analyzed using multibody dynamics tools. The impact intensity was discovered in the parameter space of the angle of the thigh, the angle of the shank, the foot strike pattern and the running speed.

The results regarding the effect of strike pattern are in coincidence with the literature: forefoot strike implies lower impact intensity and energy absorption than rearfoot strike. However, in contrast of the previous result of a two segment foot model from the related literature, the calculations indicated that the shank angle highly affects the impact intensity: the impact intensity can be reduced by foot touchdown under the hip. We showed that foot and shank cannot be analyzed in itself without considering the thigh and the total body weight, and we also confirmed that the horizontal velocity cannot be neglected when foot impact is analyzed.
\end{abstract}

Keywords

ground-foot collision, strike index, foot positioning, running form analysis, effective mass, constrained motion space kinetic energy

\section{Introduction}

Many studies like [1] and [2] help the understanding of bipedal locomotion, human walking and running. Several approaches have been developed which try to explain he dynamic background of the healthy, injury preventing, energy efficient and natural way of running [3, 4]. Many papers study the effect of foot strike pattern and footwear experimentally [5-9] and some of them find that forefoot strike running is more economical than rearfoot strike running $[10,11]$.

Energy efficiency is crucial in long distance running. The sources of energy loss are the aerodynamical forces, damping, vibrations and ground-foot collision at every footstep. Out of the above listed effects, the foot collision is quite relevant and it could be influenced by the runner by modifying his/her running form. Some tribes are naturally talented at running [12] who are capable to run extremely long distances. These people usually wear only thin sole shoes or they often run barefoot, as the people did before the invention of running shoes [7]. There is no any part in their footwear which is designed for providing flexibility and/or damping, therefore they can rely only on the flexibility of their legs and not on artificial damping which would presumably influence their body motion. The exaggeration of the convenience features of running shoes makes it hard to develop sophisticated and natural running form, which utilizes the capabilities of human leg. Although there is no proof that running in shoes causes injuries or prevents them either [5].

Jungers [5] states that there is need for more studies providing data, testable models and scientific explanation. 
Also some studies with evidence-based approach are requested to discover if there is a best footwear or best running form. Our work aims to contribute to this field by pure mechanical calculations using tools of multibody dynamics, allowing us to predict the effect of parameter variations.

Our study focuses on the effect of shank angle at landing, overstriding and foot strike pattern. These indicators are listed in [13] among such indicators of running kinematics, which can be identified using a lateral (side) view video recording of the running motion.

The evolution of our minimally complex foot impact model is presented in Section 2. Section 3 overviews the mechanical background, while Section 4 and 5 summarize the results and discussion.

\section{Mechanical models for ground-foot impact}

\subsection{Literature review}

The foot strike pattern, and kinetic and kinematical differences between barefoot and shod running were discussed in [5]. The related experimental results were interpreted by [7] using the tools of analytical mechanics. A low degree of freedom (DoF) model was introduced for investigating the effect of foot strike pattern on impact intensity and ground reaction force. The dimensionless parameter $s$ describes the foot strike pattern and is called strike index. Based on the initial contact the strike pattern is sorted into rearfoot / heel (RFS, $s=0 \ldots 0.33$ ), midfoot (MFS, $s=0.33 \ldots 0.66$ ) or forefoot (FFS, $s=0.66 \ldots 1$ ) strike (see [13]) as it is shown in Fig. 1. Here point contact is assumed, however strike index locates the centre of the pressure in reality. Fig. 2(a) shows the planar model from [7] with horizontal foot and vertical shank. The ground contact location (point $O$ ) is defined by $s$. Stiff and compliant ankle joint (point $B$ ) were considered. The pre-impact velocity was downward vertical. The authors drew the conclusion that forefoot landing provides lower impact intensity.

An extended analysis of the same mechanical model was carried out and a more algorithmic mechanical approach was applied in [14]. As Fig. 2 (b) shows, this model still

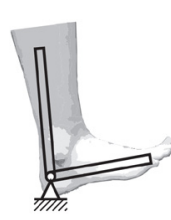

RFS

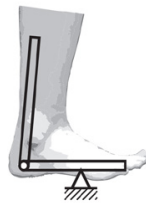

MFS

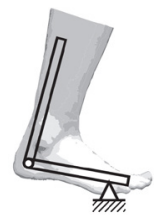

FFS
Fig. 1 Illustration of strike patterns: rearfoot (RFS), midfoot (MFS) and forefoot (FFS) strike

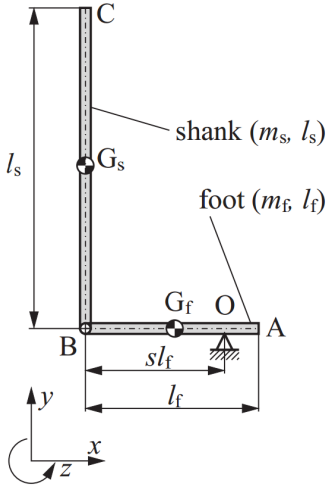

(a)

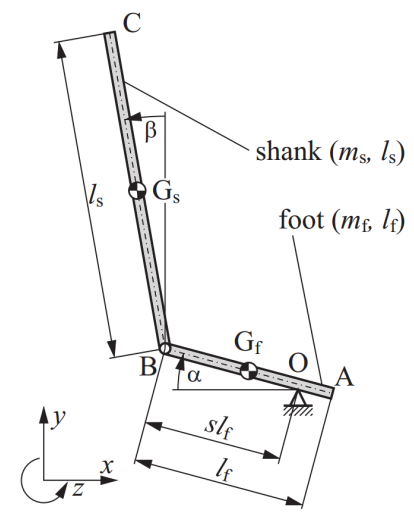

(b)
Fig. 2 (a): foot impact model presented in [7]; (b): the model introduced in [14]

involves the foot and the shank only, but foot and shank angles $(\alpha$ and $\beta$ ) are new parameters. The authors studied $\alpha$ $=0^{\circ}$ case and focused on the effect of parameter $\beta$. Besides downward vertical pre-impact velocity, the effect of horizontal velocity component was studied. The calculations are based on the consideration that ground contact is represented by geometric constraints. The kinetic energy content associated with the constrained motion, which serves as an indicator of foot impact intensity, was calculated. The authors confirmed the results of [7] and derived a conclusion that the shank angle $\beta$ which is responsible for foot positioning, does not affect the impact intensity at FFS and has limited effect at RFS. Similar results will be presented in Section 4 in the case study called "Extended knee, vertical pre-impact velocity".

\subsection{Our investigated model}

Possibly, the model shown in Fig. 2 (b) is still not detailed enough to catch the main characteristics of running style: the deduced results with respect to the shank angle $\beta$ are not in total correspondence with the empirical observations. Fig. 3 illustrates the evidence how important the shank angle $\beta$ is. When running downhill, one tries to keep the speed bounded. The breaking technique is basically the overstriding, because it results in the largest energy absorption. In contrast, a long distance road race runner, whose aim is to save energy, keeps his/her shank in negative angle before foot impact (see Fig. 3 (b)). There are definitions for overstriding, e.g. in [4] or [13]. In this article we refer to overstriding when angle $\beta$ is positive, so foot touchdown occurs in front of the body. In order to prove the effects of overstriding scientifically, we propose to accomplish a similar calculation as in [14] with a more complex but still low DoF mechanical model 


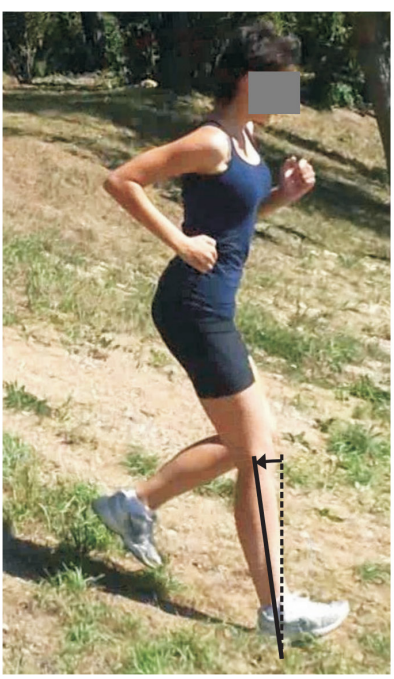

(a)

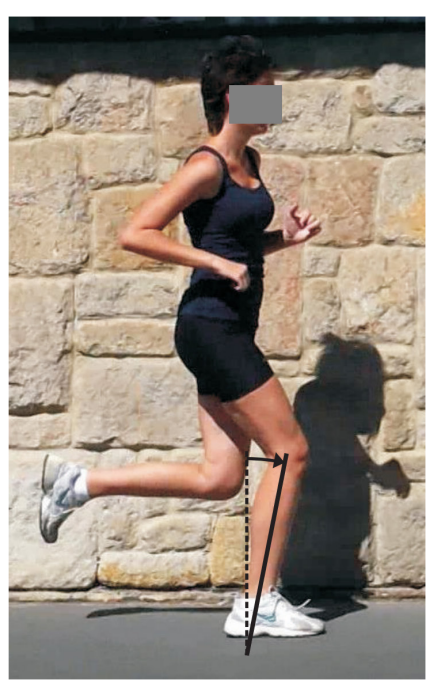

(b)
Fig. 3 Different landing strategies are used when running downhill ((a) positive shank angle) or running on at flat track ((b) negative shank angle)

like in $[9,11,15]$, which is minimally complex enough to analyze the effect of strike pattern and shank angle $\beta$ on impact intensity. Furthermore, we consider the horizontal pre-impact velocity component of the body.

The model shown in Fig. 4 contains the full leg and an additional pointmass, as in [15], where $s=0$ and $s=1$ cases were studied only (see ground-foot contact scenarios in [16]). We still focus on landing phase: the dynamic effects of strike pattern (characterized by strike index $s$ ) and foot positioning (characterized by shank angle $\beta$ ) are analysed. Our system is similar to the model in [17], however elasticity is not considered here, since the muscle forces are considered negligible comparing to impact-induced forces. Furthermore the inertia of the segments must be included to make the model able to predict impact forces.

Lieberman et al. [7] state that in case of RFS ankle compliance has little effect and there is some contribution from mass above the knee, which also encourages us to investigate an extended model which is not limited to shank and foot only, however we do not consider stiff ankle case. The other reason for focusing only on the compliant case is that the foot and the shank are connected by muscles and tendons which are flexible. The forces exerted by flexible components are negligible when impact forces arise [14].

The model shown in Fig. 4 consists of 3 segments: thigh, shank and foot joined by ideal, frictionless joints. An additional pointmass $m_{b}$ is attached to the thigh representing the mass of the trunk and the other not-modelled

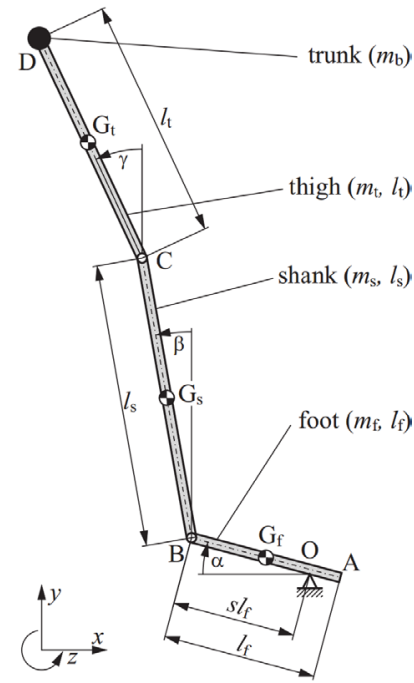

Fig. 4 The proposed minimally complex model for the investigation of ground-foot impact intensity

body parts, like head, arms and other leg. Inertial and geometric data are collected in Table 1 and adopted from [18] and [19]. The data correspond to an average 24 years old male person with $73 \mathrm{~kg}$ bodyweight and $173.1 \mathrm{~cm}$ height. Segmental centre of gravity (CoG) locations are measured from proximal end of each segment. The moment of inertia around $\mathrm{CoG}$ axis of each segment is estimated by homogeneous rod model.

The proposed 5 DoF model is described by the Cartesian coordinates of point $A$ and absolute angles $\alpha, \beta$ and $\gamma$ of the foot, shank and thigh respectively. The general coordinates are

$\boldsymbol{q}=\left[\begin{array}{lllll}x_{A} & y_{A} & \alpha & \beta & \gamma\end{array}\right]^{T}$.

The corresponding 5 by 5 mass matrix $\boldsymbol{M}$ is shown by Eq. (2). For the sake of briefness, we introduced new notations $m_{0}=m_{b}+m_{t}+m_{s}+m_{f}, m_{b t s}=m_{b}+m_{t}+m_{s}$ and $m_{b t}=m_{b}+m_{t}$, which represents merged masses of the body segments. Furthermore, $s_{\alpha}=\sin \alpha$ and $c_{\alpha}=\cos \alpha$ are introduced and the same notation is applied for $\beta$ and $\gamma$. We also introduced short notations $s_{\alpha \gamma}^{+}=\sin (\alpha+\gamma)$, $s_{\alpha \beta}^{+}=\sin (\alpha+\beta)$ and $c_{\beta \gamma}^{-}=\cos (\beta-\gamma)$.

Table 1 Inertial and geometric data of the modeled body segments: trunk $(b)$, thigh $(t)$, shank $(s)$ and foot $(f)$

\begin{tabular}{lccc}
\hline $\begin{array}{l}\text { mass, } \\
\mathrm{kg}\end{array}$ & $\begin{array}{c}\text { mass moment of } \\
\text { inertia, } \mathrm{kg} \mathrm{m}^{2}\end{array}$ & $\begin{array}{c}\text { length, } \\
\mathrm{m}\end{array}$ & $\begin{array}{c}\text { CoM position, } \\
\mathrm{m}\end{array}$ \\
\hline$m_{b}=58.5$ & - & - & - \\
$m_{t}=10.3$ & $J_{t}=13910^{-3}$ & $l_{t}=0.402$ & $d_{t}=0.164$ \\
$m_{s}=3.16$ & $J_{s}=48.210^{-3}$ & $l_{s}=0.428$ & $d_{s}=0.188$ \\
$m_{f}=1.00$ & $J_{f}=0.45610^{-3}$ & $l_{f}=0.274$ & $d_{f}=0.032$ \\
\hline
\end{tabular}


$\boldsymbol{M}=\left[\begin{array}{ccccc}m_{0} & 0 & \left(l_{f} m_{0}-d_{f} m_{f}\right) s_{\alpha} & \left(d_{s} m_{s}-l_{s} m_{b t s}\right) c_{\beta} & \left(d_{t} m_{t}-l_{t} m_{b t}\right) c_{\gamma} \\ 0 & m_{0} & \left(l_{f} m_{0}-d_{f} m_{f}\right) c_{\alpha} & \left(d_{s} m_{s}-l_{s} m_{b t s}\right) s_{\beta} & \left(d_{t} m_{t}-l_{t} m_{b t}\right) s_{\gamma} \\ \left(l_{f} m_{0}-d_{f} m_{f}\right) s_{\alpha} & \left(l_{f} m_{0}-d_{f} m_{f}\right) c_{\alpha} & J_{f}^{2}+l_{f}^{2} m_{0}-\left(2 l_{f} d_{f}-d_{f}^{2}\right) m_{f} & l_{f}\left(d_{s} m_{s}-l_{s} m_{b t s}\right) s_{\alpha \beta}^{+} & l_{f}\left(d_{t} m_{t}-l_{t} m_{b t}\right) s_{\alpha \gamma}^{+} \\ \left(d_{s} m_{s}-l_{s} m_{b t s}\right) c_{\beta} & \left(d_{s} m_{s}-l_{s} m_{b t s}\right) s_{\beta} & l_{f}\left(d_{s} m_{s}-l_{s} m_{b t s}\right) s_{\alpha \beta}^{+} & J_{s}^{2}+l_{s}^{2} m_{b t s}-\left(2 l_{s} d_{s}-d_{s}^{2}\right) m_{s} & l_{s}\left(d_{t} m_{b t}-d_{t} m_{t}\right) c_{\beta \gamma}^{-} \\ \left(d_{t} m_{t}-l_{t} m_{b t}\right) c_{\gamma} & \left(d_{t} m_{t}-l_{t} m_{b t}\right) s_{\gamma} & l_{f}\left(d_{t} m_{t}-l_{t} m_{b t}\right) s_{\alpha \gamma}^{+} & l_{s}\left(d_{t} m_{b t}-d_{t} m_{t}\right) c_{\beta \gamma}^{-} & J_{t}^{2}+l_{t}^{2} m_{b t}-\left(2 l_{t} d_{t}-d_{t}^{2}\right) m_{t}\end{array}\right]$.

In ground phase, when the foot is connected to the ground by an anchoring point, the geometric constraint vector $\varphi$ reeds:

$\boldsymbol{\varphi}=\left\lceil\begin{array}{l}x_{A}-(1-s) l_{f} c_{\alpha} \\ y_{A}+(1-s) l_{f} s_{\alpha}\end{array}\right\rceil$,

where $x_{A}$ and $y_{A}$ are the Cartesian coordinates of point $A$. The following constraint Jacobian $\Phi_{q}=\partial \varphi / \partial \varphi$ is calculated from Eq. (3) and used in later calculations, when the post-impact velocity conditions are calculated:

$\Phi_{q}=\left[\begin{array}{llllll}1 & 0 & (1-s) l_{f} s_{\alpha} & 0 & 0 \\ 0 & 1 & (1-s) l_{f} c_{\alpha} & 0 & 0\end{array}\right]$.

\section{Considerations about the mechanical description}

A feature of legged locomotion systems is the changing of topology. For instance, when ground and foot get in contact, new constraints arise and the model will have less DoF than in the airborne phase of running. Besides, foot impact with the ground is also an important phenomenon. We apply a constraint-based approach like in [14] and [20] for handling both challenges. The constraint-based approach has advantages over the method of angular momentum conservation (see [7]), which is usually applied when bipedal locomotion systems and passive dynamic walking mechanisms are analyzed, e.g. in [21] and [22]. The main strength of the constraint based approach is the applicability for closed kinematic loops (e.g. walking, when both legs touch the ground for finite time), which is not true for the approach using conservation of angular momentum [15]. Furthermore, its calculation process is more algorithmic. The constraint-based approach is explained in the followings.

\subsection{Description of impact dynamics}

The finite (continuous) dynamics and the discrete collision event (impulsive dynamics) are distinguished in the mechanical description of legged locomotion. Let the finite-time dynamics be described by the following equation of motion:

$$
\boldsymbol{M}(\boldsymbol{q}) \ddot{q}+\boldsymbol{C}(\boldsymbol{q}, \ddot{\boldsymbol{q}})=\boldsymbol{Q}(\boldsymbol{q})
$$

with vector $\boldsymbol{q}$ of general coordinates, mass matrix $\boldsymbol{M}$, and vector $\boldsymbol{C}$ of Coriolis and centrifugal terms and $\boldsymbol{Q}$ represents the non-inertial forces, like gravity.

We assume that i) the ground-foot collision is instantaneous, which leads to infinitely large instantaneous forces over infinitesimal time duration so that the net impulse due to the impact force is finite $[7,14,15]$. Secondly, ii) completely inelastic collision is also assumed, so that there is no rebound [7, 14]. Assumption iii) is that there is no slip.

Assumptions i, ii and iii lead us to consider the groundfoot contact as an instantly arising geometric constraint as in [14-16, 20, 23, 24]. Hence, equation of motion Eq. (5) can be rewritten in the following form in order to describe the impulsive dynamics [14]:

$\boldsymbol{M}(\boldsymbol{q})\left(\dot{\boldsymbol{q}}^{+}-\dot{\boldsymbol{q}}^{-}\right)=\hat{\boldsymbol{F}}(\boldsymbol{q})$,

where $\dot{\boldsymbol{q}}^{-}=\dot{\boldsymbol{q}}\left(t^{-}\right)$and $\dot{\boldsymbol{q}}^{+}=\dot{\boldsymbol{q}}\left(t^{+}\right)$are the generalized velocities right before (pre-impact) and after (post-impact) the collision respectively. $\hat{\boldsymbol{F}}$ is the net impulse of the impact forces, all other forces are neglected. Using Eq. (6), the post-impact generalized velocity vector $\dot{\boldsymbol{q}}^{+}$can be theoretically determined. While velocity condition changes instantly, the configuration $\boldsymbol{q}=\boldsymbol{q}^{+}=\boldsymbol{q}^{-}$does not change.

Before foot touchdown, the body is in flying phase, so that the system moves freely. Pose and velocity conditions are described by the generalized coordinate vector $\boldsymbol{q}$ and the pre-impact generalized velocity $\dot{\boldsymbol{q}}^{-}$. The new constraints $\varphi(q)=0$ related to the ground contact (see Eq. (3)) arise and the post-impact velocities are determined by the projection to the space of the admissible motion:

$\dot{\boldsymbol{q}}^{+}=\boldsymbol{P}_{a} \dot{\boldsymbol{q}}^{-}$,

where projection matrix $\boldsymbol{P}_{a}$ transforms into the null space of the constraint Jacobian matrix:

$\boldsymbol{P}_{a}=\boldsymbol{I}-\boldsymbol{P}_{c}$

$\boldsymbol{P}_{c}$ is the projection matrix of the constrained space:

$\boldsymbol{P}_{c}=\Phi_{q}^{\dagger} \Phi_{q}$

and the pseudo-inverse of the non-square constraint Jacobian (see Eq. (4)) can be calculated according to [25] as: 
$\Phi_{q}^{\dagger}=M^{-1} \Phi_{q}^{T}\left(\Phi_{q} M^{-1} \Phi_{q}^{T}\right)^{-1}$

Similar projection technique is presented in [16].

\subsection{Slip of the foot}

Considering a normal and convenient running, assumption iii stands, since depending on the surface quality, usually there is no significant slip of the foot in practice. However, the slip-free condition is checked, for which the geometric constraint in Eq. (3) is separated into two parts $\varphi_{t}$ and $\varphi_{n}$ constraining the tangential and the normal direction motion respectively:

$$
\varphi=\left\lceil\begin{array}{c}
\varphi_{t} \\
\varphi_{n}
\end{array}\right\rceil .
$$

For satisfying the non-slip condition, the Coulomb friction coefficient must be larger than the critical value [26]:

$$
\mu_{c}=\left|\frac{\Phi_{n} M^{-1} \Phi_{t}^{T}}{\Phi_{n} M^{-1} \Phi_{n}^{T}}\right|,
$$

where $\boldsymbol{\Phi}_{t}$ and $\boldsymbol{\Phi}_{n}$ are the Jacobians of the tangential and normal direction constraints respectively.

\subsection{A measure for the impact intensity}

The kinetic energy related to the constrained part of the motion vanishes when the foot touches the ground. This energy amount is calculated as it is done in [26]:

$$
T_{c}=\frac{1}{2}\left(\dot{\boldsymbol{q}}^{-}\right)^{T} \boldsymbol{P}_{c}^{T} \boldsymbol{M} \boldsymbol{P}_{c} \dot{\boldsymbol{q}}^{-}
$$

$T_{c}$ is called constrained motion space kinetic energy (CMSKE). Papers [14] and [20] showed that foot strike intensity can be characterised by the CMSKE which depends on the pre-impact configuration and velocity and the effective mass matrix $\boldsymbol{M}_{e}=\boldsymbol{P}_{c}^{T} \boldsymbol{M} \boldsymbol{P}_{c}$. The effective mass concept for foot impact is presented in [27] for a one DoF model. In this work the CMSKE is used for characterize the foot impact intensity: CMSKE is directly proportional to the impulse of the contact reaction force and also to the peak reaction force, as it is presented in [14] and [26].

CMSKE also can be used as an energy efficiency indicator of passive walkers [21] and [22] because their energy loss is the foot impact only.

\section{Results}

Our results cover three main cases starting from the verification of the related literature results towards more accurate modeling.

\subsection{Extended knee, vertical pre-impact velocity}

Fig. 5 shows qualitatively same result as presented in [14]. The CMSKE is calculated and then normalized by the pre-impact kinetic energy $T$. For the sake of better visibility, logarithmic scale is used in $T_{c} / T$ axis. We assume straight leg $(\beta=\gamma)$, horizontal foot $\left(\alpha=0^{\circ}\right)$ and vertical pre-impact velocity $\left(\dot{x}_{A}=0 \mathrm{~m} / \mathrm{s}, \dot{y}_{A}=-1 \mathrm{~m} / \mathrm{s}\right)$. The magnitude of the vertical pre-impact velocity is estimated based on the bouncing ball model of running [28]. The calculations confirm that shank angle $\beta$ can have a small effect on the impact intensity at low strike index: the ratio of $T_{c}$ and $T$ is $98 \%$ at $\beta=0^{\circ}$ and $92 \%$ at $\beta=15^{\circ}$. Furthermore, the impact intensity is a symmetric function of $\beta$, which does not meet with experience (see Fig. 3). Fig. 5 also confirms that larger strike index $s$ provides smaller impact and higher energy efficiency (the ratio of $T_{c}$ and $T$ is $0.25 \%$ at $s=1$ ), while the variation of $\beta$ does not have effect. The value of the effective mass $m_{e}(2,2$ element of the effective mass matrix $\boldsymbol{M}_{e}$ ) and the CMSKE is in linear relationship: $T_{c}=1 / 2 m_{e} \dot{y}_{A}^{2}$, because only one generalized velocity is nonzero out of the five. Intuition and practical observations [3] and [4] encouraged us to accomplish the following two test cases that are expected to show that the shank angle $\beta$ has much more remarkable effect on the impact intensity in reality.

It is known from experience that overstride and RFS is in relation with each other. Usually overstriding impels RFS, and it is natural that FFS occurs if the landing point is nearly below the CoG of the body. Therefore the practically relevant regions are plotted with thick curves in Figs. 5-7. While the thin lines refer to very inconvenient configurations, which typically do not come up in practice, e.g. rearfoot strike with negative $\beta$.

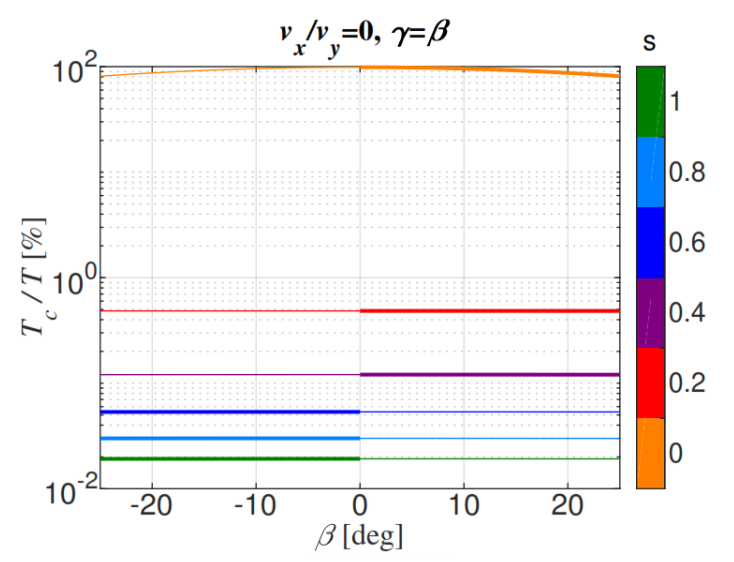

Fig. 5 CMSKE $\left(T_{c}\right)$ as function of strike index $(s)$ and shank angle $(\beta)$ in case of extended knee and vertical pre-impact velocity 


\subsection{Bent knee, vertical pre-impact velocity}

Fig. 6 shows the CMSKE in the case when the absolute angle of the thigh and the foot were set to $\gamma=15^{\circ}, \gamma=20^{\circ}$ and $\gamma=25^{\circ}$ and $\alpha=0^{\circ}$ respectively, while shank angle $\beta$ was varied in $-15^{\circ}$ to $15^{\circ}$ range. The effective mass $m_{e}$ is still in linear relationship with $T_{c}$ because only $\dot{y}_{A}$ is a nonzero pre-impact generalized velocity. The results are contradictory with [14], because $\beta$ has a remarkable effect on $T_{c}$. The worst case is rearfoot strike combined with extended knee $(\beta=\gamma)$, when almost $100 \%$ of the kinetic energy is absorbed by the impact. Overstriding (positive values of $\beta$ ) causes large energy loss and high impact, as it is expected based on practical observations. For negative values of shank angle $\beta$, the absorbed kinetic energy is less than $10 \%$. We can conclude that the results became much more realistic compared to the models shown in Fig. 2 because of involving thigh and point mass $m_{b}$ in the model. The results show that overstriding can have energy absorbing effect.

\subsection{Bent knee, tilted pre-impact velocity}

For the sake of even more realistic results, a nonzero horizontal velocity component is introduced besides the vertical one. Three different running speed values are considered: $\dot{x}_{A}=3 \mathrm{~m} / \mathrm{s}, \dot{x}_{A}=4 \mathrm{~m} / \mathrm{s}$ and $\dot{x}_{A}=5 \mathrm{~m} / \mathrm{s}$ while the vertical pre-impact velocity is still $\dot{y}_{A}=-1 \mathrm{~m} / \mathrm{s}$. The consideration of tiled pre-impact velocity was also encouraged by (reference) [9] which concludes that the foot placement and velocity is prepared well before touchdown. Fig. 7 shows that the effect of angle $\beta$ is even more substantial when rearfoot strike occurs. The constrained motion space kinetic energy is minimal, when the strike index is large and the shank angle $\beta$ is close to zero. In the best situation $2 \%$ of the pre-impact kinetic energy is absorbed only. The change of the horizontal velocity component in the relevant range (under $3 \mathrm{~m} / \mathrm{s}$ the locomotion is rather walking than running) does not affect $T_{c}$ substantially. The results of Fig. 7 change so significantly comparing to Fig. 5 and Fig. 6, because the horizontal velocity components cannot be highly influenced by the ground force which acts in vertical direction.

When considering horizontal velocity component, only $28 \%$ of kinetic energy is lost in maximum. It is because the horizontal speed component of the body does not change too much. The effective mass is not in direct connection with CMSKE, but CMSKE can be calculated as $T_{c}=1 / 2 \dot{\boldsymbol{q}}^{T} \boldsymbol{M}_{e} \dot{\boldsymbol{q}}$, which gives a linear combination of the terms of the effective mass matrix.

\section{Discussion}

The results indicate that landing just slightly in front of the $\operatorname{CoG}(\beta \approx 0)$ together with FFS $(s>0.5)$ is preferable, while overstriding $(\beta>0)$ together with RFS $(s<0.5)$ is better to be avoided, from the viewpoint of impact intensity and impact induced kinetic energy loss. Summarizing, right strike index and correct shank angle correlate, so it is indirectly confirmed that strike pattern is the most important parameter, which was drawn in [7] and [14].

Our results are in correlation with the following literature based statements of [13]. a) Runners with a RFS pattern developed more repetitive overuse injuries when compared with FFS runners. b) The flexion of the tibia helps the runner to reduce impact; therefore an extended tibia is not ideal for runners who suffer from impact-related running injuries. c) The presence and magnitude of overstriding may be the key risk factor of running injuries.

The $T_{c} / T$ ratio difference at $\beta=0^{\circ}$ and $\beta=25^{\circ}$ was $8 \%$ only, when extended knee and vertical pre-impact velocity was considered (see Fig. 5). Contrarily, our extended model and the consideration of horizontal velocity component resulted approximately $25 \%$ difference in the kinetic energy ratio $T_{c} / T$ between overstriding $\left(\beta=25^{\circ}\right)$ and landing approximately below the hip $\left(\beta=0^{\circ}\right)$ cases, as Fig. 7 shows. The impact intensity is much more sensitive to parameter $\beta$ in case of the new model. This shows that the more detailed mechanical model of the body provides substantially different results, which might be closer to the practical observations.

FFS is often followed by heel strike which causes a second impact with a second amount of absorbed kinetic energy. This phenomena was investigated in [15], however it requires more detailed analysis.

\section{Conclusion}

We accomplished purely mechanical calculations to gain information about the effect of strike pattern and foot positioning on the impact intensity and energy efficiency of running. The presented calculation is more predictive than experiments in some cases, because the effect of any parameter modification can be seen promptly.

Our enhanced model allowed us to gain a practical result that overstriding should be avoided and forefoot landing is better than heel strike both from the viewpoint of energy efficiency and injury prevention. The results show that high ground-foot impacts can be avoided purely by a proper foot positioning. In such situation not only the damping effect of the elevated and cushioned heel of the shoes protects the 

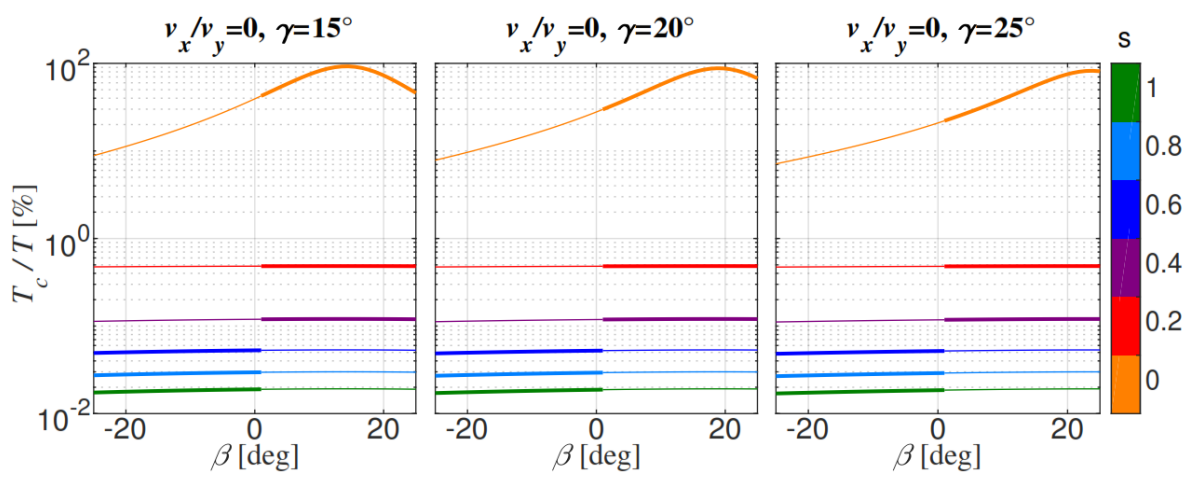

Fig. 6 CMSKE $\left(T_{c}\right)$ as function of strike index $(s)$ and shank angle $(\beta)$ in case of bent knee and vertical pre-impact velocity in different thigh angle $(\gamma)$ values
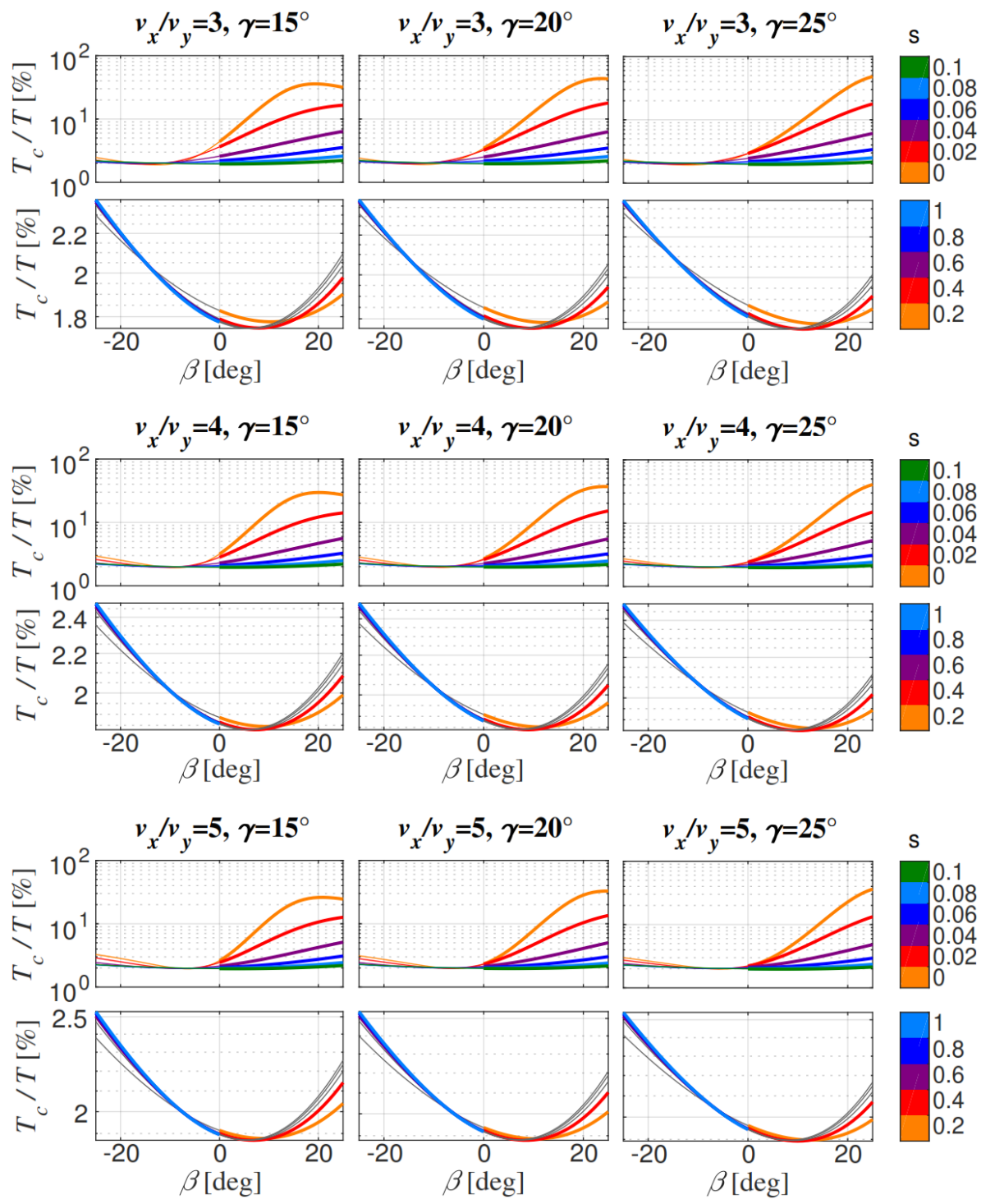

Fig. 7 CMSKE $\left(T_{c}\right)$ as function of strike index $(s)$ and shank angle $(\beta)$ in case of bent knee and tilted pre-impact velocity in different thigh angle $(\gamma)$ and horizontal velocity component $\left(v_{x}\right)$ values 
runner. This result contributes to the recent researches focus on the comparison of barefoot versus shod running which two conditions yield different ground reaction forces.

We showed that foot and shank cannot be analyzed in itself, only together with the inertia of other body parts. Also the consideration of horizontal velocity component of the body leads to more realistic results compared to the results of earlier models presented in [7] and [14]. Our results also show that the shank angle is at least as important parameter as the strike pattern. However, the practical meaning of this statement is in total correspondence with the referred literatures [5, 7] and [14] because negative shank angle impels forefoot strike.

\section{References}

[1] Blajer, W., Schiehlen, W. "Walking Without Impacts as a Motion / Force Control Problem", Journal of Dynamic Systems, Measurement, and Control, 114(4), pp. 660-665, 1992.

https://doi.org/10.1115/1.2897738

[2] Novacheck, T. F. "The biomechanics of running", Gait and Posture, 7(1), pp. 77-95, 1998.

https://doi.org/10.1016/S0966-6362(97)00038-6

[3] Dreyer, D. "Chi Running homepage", [online] Available at: www. chirunning.com [Accessed: 03 October 2018]

[4] Cucuzzella, M. "Posing the question of proper running form: natural running vs. pose method", [online] Available at: http://naturalrunningcenter.com/2013/07/30/posing-question-proper-running-form/ [Accessed: 03 October 2018]

[5] Jungers, W. L. "Barefoot running strikes back", Nature, 463(7280), pp. 433-434, 2010.

https://doi.org/10.1038/463433a

[6] Larson, P. "Comparison of foot strike patterns of barefoot and minimally shod runners in a recreational road race", Journal of Sport and Health Science, 3(2), pp. 137-142, 2014.

https://doi.org/10.1016/j.jshs.2014.03.003

[7] Lieberman, D. E., Venkadesan, M., Werbel, W. A., Daoud, A. I., D'Andrea, S., Davis, I. S., Mang'Eni, R. O., Pitsiladis, Y. "Foot strike patterns and collision forces in habitually barefoot versus shod runners", Nature, 463(7280), pp. 531-535, 2010.

https://doi.org/10.1038/nature08723

[8] Meredith, K., Castle, B., Hines, D., Oelkers, N., Peters, J., Reyes, N., Conti, C., Pollard, C., Witzke, K. "Peak Impact Ground Reaction Force during Barefoot and Shod Running", International Journal of Exercise Science: Conference Proceedings, 8(3), Article $13,2015$.

[9] Wit, B. D., Clercq, D. D., Aerts, P. "Biomechanical analysis of the stance phase during barefoot and shod running", Journal of Biomechanics, 33(3), pp. 269-278, 2000. https://doi.org/10.1016/S0021-9290(99)00192-X

[10] Gruber, A. H., Boyer, K. A., Derrick, T. R., Hamill, J. "Impact shock frequency components and attenuation in rearfoot andforefoot running", Journal of Sport and Health Science, 3(2), pp. 113-121, 2014. https://doi.org/10.1016/j.jshs.2014.03.004
The presented parameterization makes possible the extension of the mechanical model in future wok, e.g. considering the spatial dynamics, more body segments and elastic ground-foot connection. These extensions may lead to new results related to optimal upper body posture and the stabilizing and damping effect of the shoe.

\section{Acknowledgement}

Our research has been supported by the MTA-BME Research Group on Dynamics of Machines and Vehicles and the MTA-BME Lendület Human Balancing Research Group. These supports are gratefully acknowledged.

[11] Ahn, A. N., Brayton, C., Bhatia, T., Martin, P. "Muscle activity and kinematics of forefoot and rearfoot strike runners", Journal of Sport and Health Science, 3(2), pp. 102-112, 2014. https://doi.org/10.1016/j.jshs.2014.03.007

[12] McDougall, C. "Born to Run: A Hidden Tribe, Superathletes, and the Greatest Race the World Has Never Seen", 1st ed., Alfred A. Knopf, Inc., New York, USA, 2009.

[13] Souza, R. B. "An Evidence-Based Videotaped Running Biomechanics Analysis", Physical Medicine and Rehabilitation Clinics of North America, 27(1), pp. 217-236, 2016. https://doi.org/10.1016/j.pmr.2015.08.006

[14] Kövecses, J., Kovács, L. L. "2011 Symposium on Human Body Dynamics, Foot impact in different modes of running: mechanisms and energy transfer", Procedia IUTAM, 2, pp. 101-108, 2011. https://doi.org/10.1016/j.piutam.2011.04.011

[15] Zelei, A., Bencsik, L., Kovács, L. L., Stépán, G. "Energy efficient walking and running - impact dynamics based on varying geometric constraints", In: $12^{\text {th }}$ Conference on Dynamical Systems Theory and Applications, Lodz, Poland, 2013, pp. 259-270.

[16] Blajer, W., Dziewiecki, K., Mazur, Z. "An improved inverse dynamics formulation for estimation of external and internal loads during human sagittal plane movements", Computer Methods in Biomechanics and Biomedical Engineering, 18(4), pp. 362-375, 2015.

https://doi.org/10.1080/10255842.2013.799147

[17] Seyfarth, A., Günther, M., Blickhan, R. "Stable operation of an elastic three-segment leg", Biological Cybernetics, 84(5), pp. 365$382,2001$. https://doi.org/10.1007/PL00007982

[18] Dempster, W. T. "Space requirements of the seated operator", Wright Air Development Center, Air Research and Development Command, United States Air Force, Wright-Patterson Air Force Base, Dayton, Ohio, USA, WADC-technical report 55-159., 1955.

[19] Plagenhoef, S., Evans, F. G., Abdelnour, T. "Anatomical Data for Analyzing Human Motion", Research Quarterly for Exercise and Sport, 54(2), pp. 169-178, 1983. https://doi.org/10.1080/02701367.1983.10605290 
[20] Font-Llagunes, J. M., Pàmies-Vilà, R., Kövecses, J. "Configuration-dependent performance indicators for the analysis of foot impact in running gait", In: Proceedings of the ECCOMAS Thematic Conference Multibody Dynamics 2013, Zagreb, Croatia, 2013, pp. 31-32.

[21] Garcia, M., Chatterjee, A., Ruina, A., Coleman, M. "The Simplest Walking Model: Stability, Complexity, and Scaling", Journal of Biomechanical Engineering, 120(2), pp. 281-288, 1998. https://doi.org/10.1115/1.2798313

[22] McGeer, T. "Passive Dynamic Walking", The International Journal of Robotics Research, 9(2), pp. 62-82, 1990. https://doi.org/10.1177/027836499000900206

[23] Alciatore, D. G., Abraham, L. D., Barr, R. E. "Matrix solution of digitized planar human body dynamics for biomechanics laboratory instruction", In: Proceedings of the 1992 ASME International Computers in Engineering Conference and Exposition, San Francisco, USA, 1992, pp. 271-276.

[24] Yamakita, M., Asano, F. "Extended passive velocity field control with variable velocity fields for a kneed biped", Advanced Robotics, 15(2), pp. 139-168, 2001. https://doi.org/10.1163/15685530152116209
[25] Kövecses, J., Piedboeuf, J.-C., Lange, C. "Dynamic modeling and simulation of constrained robotic systems", IEEE/ASME Transactions on Mechatronics, 8(2), pp. 165-177, 2003. https://doi.org/10.1109/TMECH.2003.812827

[26] Kövecses, J., Font-Llagunes, J. M. "An Eigenvalue Problem for the Analysis of Variable Topology Mechanical Systems", ASME Journal of Computational and Nonlinear Dynamics, 4(3), pp. 031006-1-031006-9, 2009. https://doi.org/10.1115/1.3124784

[27] Chi, K.-J., Schmitt, D. "Mechanical energy and effective foot mass during impact loading of walking and running", Journal of Biomechanics, 38(7), pp. 1387-1395, 2005. https://doi.org/10.1016/j.jbiomech.2004.06.020

[28] Bencsik, L., Zelei, A. "Effects of human running cadence and experimental validation of the bouncing ball model", Mechanical Systems and Signal Processing, 89, pp. 78-87, 2017. https://doi.org/10.1016/j.ymssp.2016.08.001 